\title{
Panorama general de la relación enfermera-paciente en algunas unidades de cuidado intensivo en Bogotá
}

\author{
Diana Marcela Achury Saldaña², Luisa Fernanda Achury \\ Beltrán³, Juan Carlos Díaz Álvarez, Sandra Mónica Rodríguez \\ Colmenares5, Herly Ruth Alvarado', Consuelo Ortiz , Pilar
}

García Peñuelå, Sandra Lilian Acosta Huertas 9

doi:10.11144/Javeriana.IE16-1.pgre

Cómo citar: Achury Saldaña DM, Achury Beltrán LF, Díaz Álvarez JC, Rodríguez Colmenares SM, Alvarado HR et al. Panorama general de la relación enfermera-paciente en algunas unidades de cuidado intensivo en Bogotá. Investig Enferm. Imagen Desarr. 2014;16(1): 73-85. doi:10.11144/Javeriana.IE16-1.pgre

1. Artículo de investigación. Recibido: 15 de noviembre de 2013. Revisado: 27 de enero de 2014. Aprobado: 29 de enero de 2014.

2. Magíster en Enfermería Cardiovascular. Profesora asociada, Facultad de Enfermería, Pontificia Universidad Javeriana, Bogotá, Colombia. Coordinadora Nacional Red Internacional de Enfermería en Cuidado Crítico. Correo electrónico: dachury@javeriana.edu.co.

3. Especialista en Cuidado Crítico. Profesora instructora, Facultad de Enfermería, Pontificia Universidad Javeriana, Bogotá, Colombia. Correo electrónico: achuryl@javeriana.edu.co.

4. Doctor en Educación. Profesor asistente, Facultad de Enfermería, Pontificia Universidad Javeriana, Bogotá, Colombia. Correo electrónico: j.diaza@javeriana.edu.co.

5. Magíster en Politica Social. Profesor asistente, Facultad de Enfermería, Pontificia Universidad Javeriana, Bogotá, Colombia. Correo electrónico: javeriana.smrodriguez@javeriana. edu.co.

6. Magíster en Política Social. Profesor asistente, Facultad de Enfermería, Pontificia Universidad Javeriana, Bogotá, Colombia. Correo electrónico: javeriana.herlynga@gmail.com.

7. Magíster en Enfermería Cardiovascular. Profesora instructora, Facultad de Enfermería, Pontificia Universidad Javeriana, Bogotá, Colombia. Correo electrónico: conort02@hotmail.com.

8. Especialista en Cuidado Crítico. Profesora instructora, Facultad de Enfermería, Pontificia Universidad Javeriana, Bogotá, Colombia. Correo electrónico: pilar.garcia@javeriana.edu.co.

9. Magíster en Enfermería Pediátrica. Asesora comercial, Línea Intervencionismo Gemedco S. A. Correo electrónico: siliam10@yahoo.com. 


\section{Resumen}

Introducción: La unidad de cuidados intensivos (UCI) es un servicio de alta complejidad, cuyo objetivo es brindar un cuidado integral a aquellas personas en condiciones críticas de salud; por tal motivo, es importante contar con el recurso humano de enfermería suficiente, según la complejidad del paciente y la carga de trabajo. Una relación enfermera-paciente puede ser uno de los elementos responsables en la aparición de incidentes y eventos adversos, por lo cual es necesario conocer la situación actual de algunas UCI. Objetivo: Describir la relación enfermera-paciente en algunas UCI de Bogotá. Materiales y métodos: Para determinar la relación enfermera-paciente se aplicó una encuesta en 49 UCI de diferentes especialidades. Resultados y conclusiones: El promedio general de la relación enfermera-paciente, teniendo en cuenta todas las UCI e instituciones reportadas, corresponde a una enfermera por cada 5,98 (6) pacientes y a una auxiliar de enfermería por cada 4,59 (5) pacientes. Dadas las condiciones y el tipo de cuidado que ellos requieren, no es una relación adecuada si se compara con estándares internacionales; sin embargo, es imposible confrontarlo con estándares nacionales, porque Colombia no cuenta con lineamientos cuantitativos, aunque sî posee parámetros cualitativos respaldados por las leyes 911 y 266, así como algunos estudios que demuestran la necesidad de aplicar e interpretar adecuadamente herramientas de medición de carga de trabajo, de tal manera que se pueda establecer y sustentar, de la manera más objetiva posible, el número de pacientes por enfermera.

Palabras clave: relación enfermera-paciente, enfermería, carga de trabajo, cuidado intensivo.

Palabras clave descriptores: relaciones enfermero-paciente, enfermería, carga de trabajo, cuidados intensivos.

\section{Overview of the nurse-patient relationship in some intensive care units in Bogotá}

\section{Abstract}

Introduction: The intensive care unit (ICU) is a high-complexity service, which aims to provide comprehensive care to people in critical health conditions; for this reason, it is important to have sufficient nursing human resources according to the patient complexity and the workload. A nurse-patient relationship can be one of the responsible elements for the occurrence of incidents and adverse events, which means it is necessary to know the current status of some ICU. Objective: To describe the nurse-patient relationship in some ICU of Bogota. Materials and Methods: To determine the nursepatient relation a survey was applied in 49 different ICU specialties. Results and Conclusions: The overall average of nurse-patient relationship, taking into account all ICU and reported institutions corresponds to one nurse per every 5.98 (6) patients and a nurse's aide per every 4.59 (5) patients. Given the conditions and the type of care they require it is not a suitable ratio when compared with international standards; however, it is impossible to compare this with national standards, because Colombia does not have quantitative guidelines, although it has qualitative parameters backed by laws 911 and 266, as well as some studies that demonstrate the need for application and proper interpretation of measurement tools of the workload, so the number of patients per nurse can be established and sustained, as objectively as possible.

Keywords: nurse-patient relationship, nursing, workload, intensive care. 


\section{Panorama geral da relação enfermeiro-paciente em algumas unidades de tratamento intensivo em Bogotá}

\section{Resumo}

Introdução: A unidade de tratamento intensivo (UTI) é um serviço de alta complexidade que visa brindar atendimento integral para pessoas em condições críticas de saúde; por tal razão, é importante contar com suficiente recurso humano de enfermagem, de acordo à acuidade do paciente e a carga de trabalho. A relação enfermeiro-paciente pode ser um elemento responsável pela ocorrência de incidentes e eventos adversos, pelo qual é preciso conhecer a situação atual de algumas UTI. Objetivo: Descrever a relação enfermeiro-paciente em algumas UTI de Bogotá. Materiais e métodos: Para determinar a relação enfermeiro-paciente foi aplicado um inquérito a 49 UTI de diferentes especialidades. Resultados e conclusões: A média geral da relação enfermeiro-paciente, considerando todas as UTI e instituições reportadas, corresponde a um enfermeiro por cada 5,98 (6) pacientes e um auxiliar de enfermagem por cada 4,59 (5) pacientes. Dadas as condições e tipo de cuidado que eles requerem, não é uma relação adequada se comparado com padrões internacionais; no entanto, é impossivel confrontá-lo com padrões nacionais porque Colômbia não tem diretrizes quantitativas, embora tenha parâmetros qualitativos respaldados pelas leis 911 e 266, assim como alguns estudos que demostram a necessidade de aplicar e interpretar adequadamente ferramentas de aferição de carga de trabalho, de maneira tal de puder estabelecer e sustentar, do jeito mais objetivo possivel, o número de pacientes por enfermeiro.

Palavras-chave: relação enfermeiro-paciente, enfermagem, carga de trabalho, tratamento intensivo.

Palavras chave descriptor: relações enfermeiro-paciente, enfermagem, carga de trabalho, terapia intensiva. 


\section{Introducción}

La Red Internacional de Enfermería en Cuidado Crítico (Reinecc) es una estrategia de trabajo y cooperación que convoca a diferentes profesionales de enfermería que laboran en el área de cuidado crítico, tanto en el ámbito académico como en el hospitalario, y se orienta al fortalecimiento de la calidad de cuidado en el paciente adulto y pediátrico. Uno de los aspectos que ha venido analizando el grupo en torno a la calidad de cuidado es la relación enfermera-paciente, dado que en Colombia, a diferencia de los otros países, no se cuenta con una información precisa y relaciones homogéneas del número de profesionales de enfermería y el paciente.

Además, la relación enfermera-paciente se considera una de las herramientas de la gestión del cuidado y se convierte en un aspecto que ha generado polémica durante muchos años, puesto que cuando se habla de este, inmediatamente se traduce en sobrecarga laboral. Algunos estudios han detectado que la sobrecarga laboral no solo depende del número de enfermeras, sino de otras variables, como la carga de trabajo, el entorno laboral, la complejidad del paciente, la capacidad del personal de enfermería, etcétera (1). En Colombia, la Ley 911 del 2004 (2), en su artículo 5, capítulo 2 , señala que:

El profesional de enfermería solamente podrá responder por el cuidado directo de enfermería, cuando la relación de número de personas asignadas para que sean cuidadas por el profesional de enfermería, con la participación del personal auxiliar, tenga en cuenta la complejidad de la situación de salud de las personas y sea tal que disminuya posibles riesgos, permita cumplir con los estándares de calidad y la oportunidad del cuidado.

Por otra parte, la Ley 266 de 1996 (3), en su artículo 19, numeral 5, instituye que "Las disposiciones para el cálculo de personal de enfermería, estarán basadas en normas nacionales e internacionales, que tengan en cuenta el estado de salud de los usuarios que demanden mayor o menor tiempo de atención de enfermería".

En la Resolución 1441 del 2013 (4) se establecen los procedimientos y las condiciones que deben cumplir los prestadores de servicios de salud para habilitar los servicios; además, se hace evidente la necesidad de contar con un recurso humano cualificado, pero no se encuentran pautas estandarizadas de relación enfermera-paciente. Con lo anterior se comprueba la existencia de pautas generales relacionadas con la relación enfermerapaciente; no obstante, es necesario que el profesional de enfermería pueda operacionalizarlas en la práctica, mediante la aplicación de instrumentos que permitan determinar la relación enfermera-paciente de forma cuantitativa, según el perfil del paciente, los cuidados de enfermería requeridos y la complejidad del tratamiento médico.

En la actualidad existen diferentes escalas que establecen tal relación, desarrolladas desde los inicios de la década de los setenta, cuando 
los investigadores comenzaron a dedicar gran parte de sus esfuerzos a la creación de sistemas capaces de medir de forma objetiva, reproducible, fiable y sensible las actividades del personal dedicado a la atención de pacientes (5). Cullen y Cyvetta, en 1974, crearon el sistema de puntuación Therapeutic Intervention Scoring System (TISS), que constaba de 76 items, con el objetivo de medir el esfuerzo asistencial de enfermería (6).

Sin embargo, en 1996, en Europa se desarrolló y validó una versión simplificada, el TISS-28, cuyos autores concluyeron que podía reemplazar la versión original en la práctica clínica de la terapia intensiva (7). Este formato tipo encuesta consta de siete apartados tipo Likert, cada uno de ellos con varias actividades a las cuales se les asigna un puntaje (de 1 a 8: a mayor complejidad mayor puntaje), de acuerdo con la complejidad de la intervención terapéutica aplicada por el personal de enfermeria, para luego sumar las actividades realizadas.

El puntaje final del TISS-28 varía de un mínimo de 0 a un máximo de 76 puntos, porque clasifica a cada paciente en una de cuatro clases: I, II, III y IV. De acuerdo con Sánchez (8), los pacientes clase I, con menos de 10 puntos, no requieren terapia intensiva o solo necesitan observación, por lo que precisan una relación enfermera-paciente 1:4 $(0,25)$. Los enfermos de clase II (10-19 puntos) son aquellos que pueden manejarse con una razón $1: 4(0,25)$ por una enfermera especialista. Los pacientes clase III son los estables, con 20-39 puntos, y pueden ser atendidos junto con un enfermo clase II en una razón 1:2 $(0,5)$ y de estar inestables, pueden requerir una razón 1:1. Finalmente, los clase IV, con 40 o más puntos, necesitan una relación $1: 1$ (1) o 2:1 (9).

Dado que el TISS es una escala que ha presentado dificultades en su aplicación, debido a su extensión, se desarrolló un instrumento que resume en 9 indicadores los 28 items del TISS, denominado Nine Equivalents of Nursing Manpower Usescore (NEMS), el cual mide las cargas de trabajo del personal de enfermería (10).

De forma adicional, otra escala que se ha venido utilizando es Nursing Activity Score (NAS) (11), que expresa el porcentaje de tiempo de trabajo de enfermería que requiere la atención de cada paciente, de manera que una suma de 100 puntos representa el total de tiempo que una enfermera dedica al paciente crítico por turno. No obstante, es más compleja de aplicar, motivo por el cual, en los estudios realizados, con mayor frecuencia es utilizado el TISS y el NEMS.

Finalmente, es importante mencionar que una asignación inadecuada del profesional de enfermería no solo incide de manera negativa en los resultados obtenidos en los pacientes, sino que también afecta a las enfermeras, quienes corren un riesgo mayor de fatiga emocional, estrés, insatisfacción en el trabajo y agotamiento. De esta manera, el artículo busca dar a conocer un panorama general de la relación enfermerapaciente en algunas unidades de cuidado intensivo de Bogotá, con el propósito de tener un insumo que les permita a los profesionales ajustar dicha relación. 


\section{Materiales y métodos}

Se realizó un diagnóstico situacional de la relación enfermera-paciente en 19 instituciones que ofrecen atención especializada en unidades de cuidado intensivo en la ciudad de Bogotá. Se aplicó una encuesta diseñada por las autoras, que constaba de 5 preguntas relacionadas con el número de unidades de cuidado intensivo por área geográfica, tipo de unidad, relación enfermera-paciente y complejidad de los pacientes. La encuesta se envió por internet a 19 profesionales de enfermería que hacen parte de la Reinecc, capítulo Colombia, que voluntariamente quisieron participar. La información recolectada se organizó y procesó en cuadros descriptivos en Excel ${ }^{\circledR}$, versión 2010. Para la presentación de resultados se utilizó estadística descriptiva.

\section{Resultados}

El presente diagnóstico situacional sobre la relación enfermera-paciente se sustenta en la consulta hecha a 49 unidades que ofrecen servicios de cuidado intensivo con diferentes especialidades, distribuidas en 19 instituciones.

En el consolidado general de la relación enfermera-paciente de las unidades de cuidado intensivo participantes (datos tabla 1) se puede observar que 19 instituciones reportaron que en las 49 unidades de cuidado intensivo se tienen disponibles 504 camas, distribuidas así: 302 camas en 27 unidades de cuidado intensivo adulto, 50 camas en 5 unidades pediátricas, 75 camas en 7 unidades para cuidado intensivo neonatal, 53 camas en 5 unidades para neonatos intermedios y 24 camas en 5 unidades para cuidado intensivo intermedio adulto. En las unidades de cuidado intensivo adulto, la relación enfermera profesional-paciente es de 1:4,8; mientras que la relación auxiliar de enfermería-paciente es de 1:3. En pediatría, la relación enfermera profesional-paciente es $1: 5,8$, y la relación auxiliar de enfermería-paciente, de 1:5. En neonatos, la relación enfermera profesional-paciente es 1:6,1 y la de auxiliar de enfermeríapaciente es 1:5,5. En las unidades de cuidado intermedio adulto, la relación enfermera profesional-paciente es 1:6,4 y la relación auxiliar de enfermeria-paciente es 1:3,2. En unidades de cuidado intermedio neonatal, la relación enfermera profesional-paciente es 1:6,8, y la de la auxiliar de enfermería-paciente, 1:6,25.

El promedio general de la relación enfermera profesional-paciente, teniendo en cuenta todas las unidades de cuidado intensivo e instituciones reportadas, corresponde a una enfermera por cada 5,98 (6) pacientes y de una auxiliar de enfermería por cada 4,59 (5) pacientes. La complejidad de las unidades de cuidado intensivo reportada fue de alta y media; sin embargo, no todas las unidades participantes en este estudio informaron el uso de escalas para determinarla, por lo que no se establecieron objetivamente las características de la complejidad de estas unidades. 


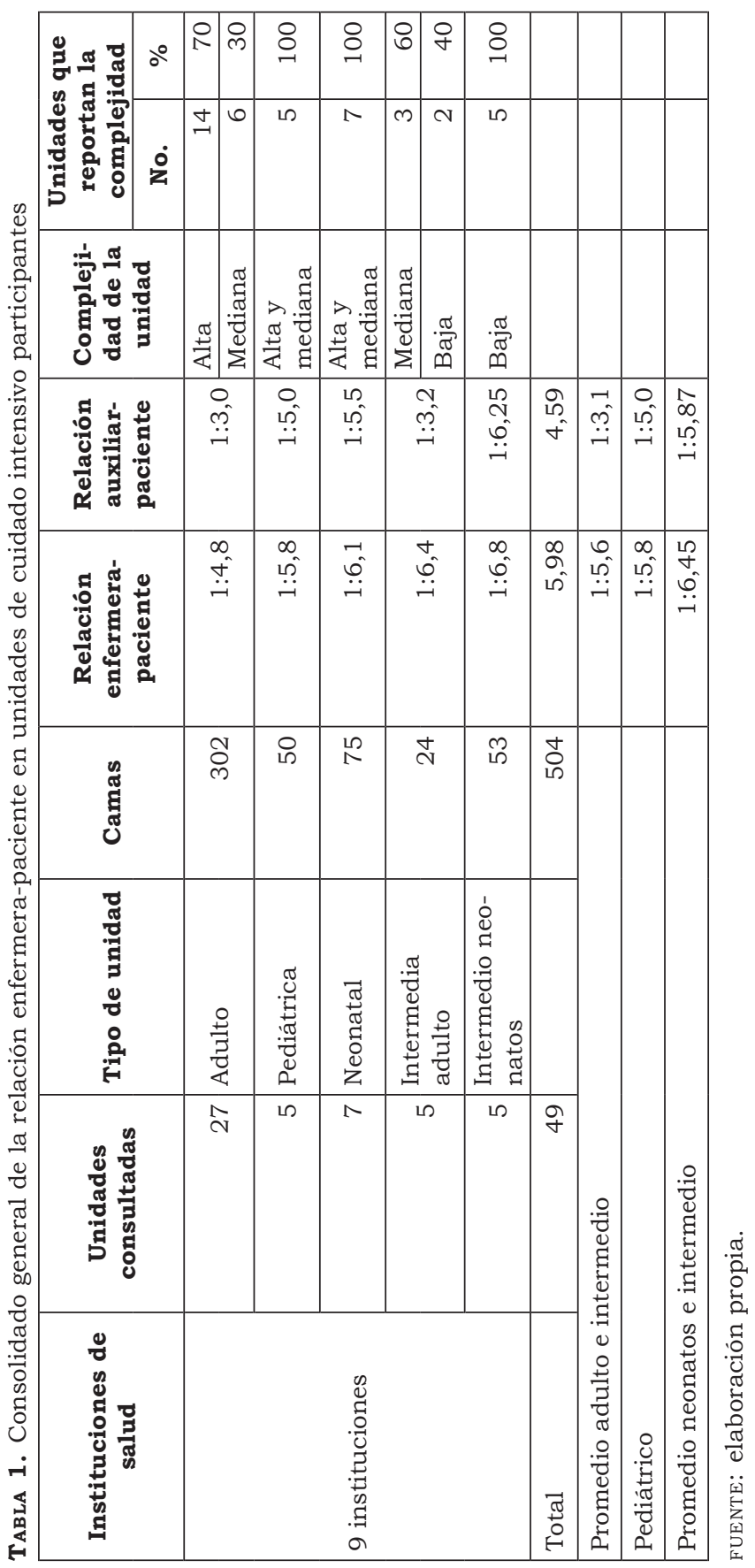




\section{Discusión}

En el presente estudio diagnóstico, el promedio general de la relación enfermera profesional-paciente en 49 unidades de cuidado intensivo e intermedio, cuyas especialidades corresponden a la atención y cuidado del paciente critico adulto, pediátrico y neonatal, correspondió a 5,98 pacientes, y en relación con el auxiliar de enfermería fue de 4,59 pacientes. Estos resultados fueron interpretados por comparación con estándares internacionales en sistemas de clasificación de complejidad (gravedad y número de intervenciones terapéuticas).

En el mundo predomina la relación enfermera-paciente 1:1, debido a las características de los sistemas de salud de los países desarrollados como Estados Unidos, Reino Unido y Australia, y a los niveles de formación para enfermeria, entre otros factores, que influyen para que la relación enfermera-paciente sea allí menor en comparación con Colombia; además, la formación de las enfermeras en estos países exige diferentes niveles tanto de conocimiento como de preparación: algunas ejecutan el cuidado directo como la enfermera registrada, quien se ha preparado académicamente durante cuatro o cinco años. Por su parte, con frecuencia, las enfermeras que realizan práctica avanzada desarrollan actividades similares a las de los médicos y requieren una formación adicional de dos años con dedicación de tiempo completo, de acuerdo con la especialidad seleccionada (12).

Lo encontrado en este estudio diagnóstico difiere significativamente de la relación enfermera-paciente internacional. La mayoría de las unidades de cuidado intensivo en Colombia no tienen estandarizados sistemas de medición que permitan a los profesionales determinar la carga de trabajo de enfermería y cuantificarla a partir de los cuidados $(13,14)$; igualmente, la formación del profesional de enfermería en Colombia tiende a cualificarse y tecnificarse con formación posgradual, a partir de estudios de especialización, maestría y doctorado. El pregrado dura cuatro o cinco y un posgrado, de uno a cinco; sin embargo, en las unidades de cuidado intensivo laboran enfermeras con experiencia y con formación de especialista, sus funciones asistenciales no se diferencian y se cuenta con auxiliares de enfermeria, quienes apoyan el cuidado de los pacientes mediante actividades de bienestar y confort.

Los estudios nacionales e internacionales sobre eventos adversos describen la relevancia del factor humano como un determinante para su presentación. Estos señalan que la carencia o insuficiencia de personal asistencial en contextos de atención de salud alcanza proporciones críticas, lo que da lugar a un significativo incremento en las estancias hospitalarias y en la morbimortalidad de los pacientes afectados por eventos adversos que pueden evitarse (14). Las enfermeras corren mayores riesgos de presentar fatiga emocional, estrés, insatisfacción en el trabajo y agotamiento.

La situación descrita muestra que es necesaria la estandarización de los instrumentos o escalas, dentro de los que se resalta la aplicación del TISS28 y el NEMS, que permiten identificar la existencia de sobrecarga laboral, la relación enfermera-paciente ideal y el número de intervenciones de cuidado.

Dentro de los estudios que utilizan el TISS-28 para respaldar la relación enfermera-paciente se puede mencionar el de Díaz y colaboradores 
(15), que respalda esta relación con el uso del TISS-28, pues señala que existe un déficit de enfermeras del 2,6 (33\%) para atender en promedio a 9,9 pacientes, de los cuales 3,8 corresponden a pacientes clase IV, y 6, a clase III.

Rodríguez y colaboradores (16) concluyeron en su estudio que una asignación enfermera-paciente, basada en la evaluación de la complejidad clínica por medio del instrumento TISS-28, disminuye los dias de estancia hospitalaria en la unidad de cuidado intensivo y promueve una recuperación más rápida de los pacientes.

Pazetto (17) informa que la aplicación sistemática del TISS-28 ayuda a determinar la relación de los profesionales de enfermería ideal, para garantizar la mejor atención posible a los pacientes y la oportunidad de presentar los resultados obtenidos objetivamente. No obstante, a pesar de los resultados que demuestran la utilidad del TISS-28, en términos de la determinación de la relación enfermera-paciente, en Colombia la escala solo se utiliza para clasificar al paciente de acuerdo con el tipo de cuidado, sea intensivo o intermedio, y no para determinar la relación enfermerapaciente según la clase I, II, III y IV, uso para el que fue construido, lo cual determina su subutilización.

Al igual que el TISS-28, el NEMS es otra escala que ha permitido determinar de forma sencilla las cargas de trabajo para enfermería. De acuerdo con lo reportado por Barroso y colaboradores (9), cada grupo de pacientes, sean estos poscirugía cardiaca, coronario, politraumatizado, médico polivalente o postoperatorio de cirugía, representa una carga de trabajo distinto; sin embargo, tales cargas no presentan diferencia en la ejecución del cuidado en los tres turnos (mañana, tarde y noche).

Teniendo en cuenta que el comportamiento de cuidado en adulto, pediatría y neonato tiene variaciones en la relación enfermera-paciente, es necesario diferenciar estos promedios. En pediatria encontramos que la relación enfermera-paciente es 1:5,8, y la relación auxiliar-paciente es de 1:5. Para neonatos la relación enfermera-paciente es $1: 6,1$, y la relación auxiliar-paciente es de 1:5,5. En intermedios neonatos, la relación enfermera-paciente es 1:6,8, y la relación auxiliar-paciente es de 1:6,2. En este aspecto es imprescindible mencionar que, al ser la anatomía y fisiología del niño y del neonato diferentes a la del paciente adulto, las necesidades y los cuidados que requieren los individuos de estos grupos poblacionales son distintos en todo sentido, en la medida que dichas condiciones los hacen más susceptibles de presentar eventos adversos; por ello se precisa de una relación enfermera-paciente óptima y capaz de cumplir a cabalidad con los requerimientos de la población. Con base en ello es posible afirmar que el profesional de enfermería que atiende al paciente pediátrico necesita, adicionalmente, un entrenamiento especial que le permita - sustentado en un amplio conocimiento- satisfacer todas aquellas necesidades que niño no puede realizar por sí solo, aspecto en el cual la vinculación de la familia y un cuidado multidisciplinar son indispensables.

En términos de escalas de medición, tanto el TISS como el NEMS han sido ampliamente utilizados en la población adulta; no obstante, un 
estudio realizado en el 2013 encontró que estas dos escalas son válidas en poblaciones pediátricas, pues identifican la gravedad de las condiciones del paciente, las intervenciones terapéuticas y los requerimientos de cuidado de enfermería (18). Sin embargo, al relacionar los datos encontrados en el presente diagnóstico situacional, no se encontraron estudios nacionales que determinen la relación ideal enfermera-paciente pediátrico en las unidades de cuidado intensivo; mientras que un estudio realizado en Estados Unidos por Ream (19) encontró una relación enfermera-paciente de 1:1,3 $\pm 0,2$, así como una relación directamente proporcional entre las extubaciones no programadas y el incremento del número de pacientes a cargo de una enfermera.

De acuerdo con lo reportado en el presente diagnóstico, las cifras de relación enfermera-paciente sobrepasan lo establecido en los estándares internacionales, por lo que es importante mencionar que en las unidades de cuidado intensivo pediátrico participantes no se utilizan con frecuencia las escalas para determinar esta relación, lo que hace imprescindible una mayor investigación sobre el tema.

En neonatos, los enfoques para asignar las enfermeras a unidades de cuidado intensivo deben sustentarse en las funciones del servicio ofrecido. Recomiendan la presencia de una enfermera profesional por cada dos niños en incubadoras y de un auxiliar de enfermería por cada cuatro (20).

Según lo descrito en las cifras reportadas del presente diagnóstico, la relación enfermera-paciente supera estas cifras, y su incremento es de siete pacientes más para la enfermera profesional. Sin embargo, de acuerdo con lo reportado, no se tiene claridad sobre si todas las camas neonatales manejan niños que requieren cuidado intermedio, lo cual limita la presente discusión.

En un estudio de la Universidad de La Sabana, en 2006, se determinó que en las unidades pediátricas y neonatales, la relación enfermerapaciente era de 1:7, y la de auxiliar de enfermería de 1:3; adicionalmente, las enfermeras tendian a realizar actividades administrativas, y las auxiliares, atención directa (21).

A pesar de esto, esta relación enfermera-paciente tampoco parece estar sustentada en la aplicación del Neonatal Therapeutic Intervention Scoring System, el cual se fundamenta en la cantidad de cuidados suministrados al paciente. Según García de Lorenzo, sus resultados no solo cuantifican el empleo de recursos, sino que también tiene implicaciones para estimar la gravedad de los pacientes (22).

Finalmente, algunos autores, como Moreno y colaboradores (23), con el ánimo de garantizar una adecuada relación enfermera-paciente, de forma adicional al uso de los instrumentos mencionados, describieron la siguiente fórmula:

Número de camas asistidas $=[\mathrm{A} \times \mathrm{B} \times \mathrm{C} \times \mathrm{D} \times \mathrm{E}] /[\mathrm{F} \times \mathrm{G}]$

Donde:

A: número de turnos por día (habitualmente 3). 
B: número de camas de la unidad.

C: número de días que la unidad trabaja a la semana (habitualmente 7).

D: tasa de ocupación; varía para cada unidad pero habitualmente es del $85 \%$.

E: trabajo adicional por días festivos, enfermedad, etc.

F: número de enfermeras en la unidad de cuidado intensivo.

G: número de días que cada profesional está trabajando por semana (habitualmente 5).

Con lo anterior se hace imprescindible que los profesionales de enfermería usen tanto de las escalas como de la fórmula reportada por Moreno, con el propósito de objetivar el cálculo de la relación-enfermera paciente y así impactar de forma positiva y relevante la calidad del cuidado, lo que se asocia con una reducción en la presentación de eventos adversos e incidentes, disminución de los dias de estancia hospitalaria e incremento en la recuperación de los pacientes.

\section{Conclusiones}

La relación enfermera-paciente correspondió a 1:6, la cual —dadas las condiciones y el tipo de cuidado que requieren los pacientes - no es considerada una relación adecuada si se compara con estándares internacionales; adicionalmente, en Colombia no existen estándares cuantitativos que permitan hacer esta correlación, por lo que la distribución enfermerapaciente se sustenta en lineamientos cualitativos respaldados por ley. Por esta razón, algunos estudios sustentan la necesidad de aplicar e interpretar adecuadamente dichas herramientas de medición de carga de trabajo, de forma tal que permitan, de forma objetiva, establecer la relación enfermerapaciente requerida.

Para poder estandarizar la relación enfermera-paciente en las unidades de cuidado intensivo es necesario aplicar e interpretar las escalas TISS-28 y NEMS para la población adulta y pediátrica, el Neonatal Therapeutic Intervention Scoring System para los neonatos, así como la fórmula propuesta por Moreno y colaboradores, de tal manera que se pueda determinar de forma más objetiva la relación enfermera-paciente requerida según las condiciones particulares de los pacientes.

\section{Referencias}

1. Amaro T. Relación ratio enfermera/enfermo y la experiencia profesional con los eventos adversos de los cuidados enfermeros: caídas, uso indebido del pañal y úlceras por presión. Reduca. 2009;1(2):22-34.

2. Ley $911 / 2004$, responsabilidad deontológica para el ejercicio de la profesión de enfermería en Colombia.

3. Ley 266/1996, reglamentación de enfermería en Colombia.

4. Resolución 1441/2013 de 6 de mayo, por la cual se definen los procedimientos y condiciones que deben cumplir los prestadores de 
servicios de salud para habilitar los servicios y se dictan otras disposiciones. Ministerio de Salud y Protección Social de Colombia.

5. Romero-Massa E, Lorduy-Bolivar J, Pájaro-Melgar C, Pérez-Duque C. Relación entre la carga laboral de enfermería y la gravedad del paciente en unidades de cuidado intensivo de adultos. Aquichán. $2011 ; 11(2): 173-86$.

6. Abizanda R, Jordá R, Valle FJ et al. Niveles de asistencia en una UCI: delimitación mediante la aplicación racionalizada del sistema TISS. Med Intensiva. 1983;7:272-88.

7. Reis Miranda D, Rijk de A, Schaufeli W. Simplified Therapeutic Intervention Scoring System: The TISS-28 items. Results from a multicenter study. Crit Care Med. 1996;24:64-73.

8. Sánchez L, Reyes M, Ector M, González A, Magdaleno M, Gonzales M. Validación del sistema simplificado de calificación de la intervención terapéutica (TISS-28) en población mexicana: estudio multicéntrico. Rev Mex de Med Crítica. 2000;14(6):191-6.

9. Barroso A, Fuente Juárez AI, López Cid JJ, Millán Vásquez FJ, Rosado Muñoz N, Simón García MJ et al. Análisis del uso de los recursos humanos enfermeros en una unidad de cuidados intensivos polivalente: situación con el resto de UCIs europeas. Enferm Intensiva. 2001;12(3):127-34.

10. Reis MD, Moreno R, Iapichino G. Nine equivalents of nursing manpower use score (NEMS). Intensive Care Med. 1997;23:760-65.

11. Bernat A, Abizanda Campos R, Cubedo Rey M, Quintana Bellmunt J, Sanahuja Rochera E, Sanchís Muñoz J et al. Nursing Activity Score (NAS) Nuestra experiencia con un sistema de cómputo de cargas de enfermería basado en tiempos. Enferm Intensiva. 2005;16(4):164-73.

12. NP Central. Nurse Practicioner FAQ or "How to become a nurse practitioner" [internet]. [Acceso 2013 nov]. Disponible: http://www.npcentral.net/consumer/npfaq.shtml.

13. Velandia AL. Regimenes laborales en enfermería y calidad de atención. Avances en Enfermería. 2002;20(2):5-22.

14. Ministerio de Sanidad y Consumo. Estudio Nacional de Efectos Adversos Ligados a la Hospitalización: ENEAS 2005 [internet]. [Acceso: 2013 nov]. Disponible en: http:/ / www.errorenmedicina.anm.edu.ar/ pdf/recursos/documentos/43_estudio_ENEAS.pdf .

15. Diaz A, Begustain A, Fernández N, Azcúnaga MF (col.). Relación paciente-enfermería a través de la evaluación clínica del sistema Therapeutic Intervention Scoring System (TISS) 28. Rev Urug de Enferm. 2006;1(2):1-11.

16. Rodríguez L, Villa C, Libreros B, Bonilla L. Efectos de la asignación enfermera paciente a través de la evaluación clínica (TISS-28). Rev Enferm IMSS. 2002;10(2):73-8.

17. Pazzeto A, Sueko S, Zanei V, Yamaguchi I. Carga de trabalho de enfermagem e sua relação com a gravidade dos pacientes cirúrgicos em UTI. Acta Paul Enferm. 2006;19(1):16-20. 
18. Cantabro ST, Stochero K, Eidt OR, Piva JP, Ramos García P. Concurrent validation of nursing scores (The NEMS and TISS-28) in pediatric intensive care. Acta Paul Enferm. 2013;26(2):123-9.

19. Ream R, Mackey K, Leet T, Green MC, Andreone TL, Loftis LL, Lynch RE. Associations of Nursing workload and unplanned extubations in a pediatric intensive care unit. Pediatr Crit Care Med. 2007 Jul;8(4):366-71.

20. Amaya de Gamarra I, Suárez de Betancourt M, Villamizar Carbajal B. Cuidado de enfermería al neonato crítico. Guías ACOFAEN [internet]. Biblioteca Las Casas; 2005. Disponible en: http://www.index-f. com/lascasas/documentos/1c0023.pdf.

21. Castro JH, Castro SY, Rodríguez CC. Propuesta de un sistema de registro de las intervenciones de enfermería en cuidado intensivo pediátrico y neonatal, basado en el modelo de adaptación de Callixta Roy [trabajo de grado en internet]; 2007. Disponible en: http://intellectum.unisabana.edu.co:8080/jspui/bitstream/10818/2412/1/121869.pdf.

22. García de Lorenzo A. Scores pronósticos y criterios diagnósticos en el paciente crítico. 2a ed. s. 1.: Ergon; 2006.

23. Cervantes M. Uso de recursos y su correlación con la gravedad de la enfermedad en la terapia intensiva. Revista de la Asociación Mexicana de Medicina Crítica y Terapia Intensiva. 2007;11(4):164-9. 
